\title{
THE DETECTION OF IONIZED HELIUM \\ AND CARBON IN THE PULSATING \\ DB DEGENERATE GD358
}

\author{
Edward M. Sion \\ Department of Astronomy and Astrophysics \\ Villanova University \\ James Liebert \\ Steward observatory \\ University of Arizona \\ Gerard Vauclair \\ Observatoire de Toulouse \\ France \\ Gary Wegner \\ Department of Physics and Astronomy \\ Dartmouth College
}

\section{Introduction}

Spectroscopic observations of hot white dwarfs utilizing the Explorer (IUE) high resolution spectrograph have led to the important discovery of ion absorption features (undetectable at low resolution) which have been ascribed to wind outflow in some cases (cf. Bruhweiler and Kondo 1983) and formation at the photosphere (cf. Sion and Guinan 1983; Bruhweiler and Kondo 1983; Dupree and Raymond 1982) in others. These line features, often weak and sharp, have presented a fundamental challenge to current understanding of the complex interplay of physical processes which control observed surface abundances in white dwarfs: gravitational/thermal diffusion, selective radiative support of ions, mass loss, convective dilution and mixing, and accretion (cf. the review by Vauclair, this volume and references therein).

Unfortunately IUE echelle observations have been possible only for the brightest dozen or so hot white dwarfs of spectral types DA, DAO, and DO, due to the exceedingly long exposure times required. Until the observations reported in this paper, IUE echelle data for non-DA stars was restricted to objects with Teff $>47000 \mathrm{~K}$. Moreover, since no high resolution IUE observation had ever been attempted for $a$ DB, one of us (E.M.S.) proposed that at least one IUE echelle spectrum of a DB should be part of the IUE archives and might reveal some surprises, following the precedence set by other unexpected detections in the IUE echelle spectra of hot white dwarfs. Arrangements for a joint Us-European exposure and choice of targets were made by one of us (J.L.). As a result we have therefore extended our IUE echelle observations downward 
in temperature to the essentially pure helium composition DB white dwarfs by combining back to back US and European low background IUE observing shifts. We have selected as the most optimum target, GD358, prototype of the class of six known pulsating DB stars and one of the hottest DB stars. Our objectives were: (1) to search for any evidence of metals in the photosphere (2) search for possible mass outflow; (3) place stringent constraints on the carbon abundance in a hot DB; and (4) look for evidence of absorption wings at Lyman alpha + He II (1215A). The results of this search are presented below.

II. Observations

Two separate high resolution IUE spectra of GD358 were obtained during the 10 th and 11 th observing episodes. The first image SWP31432, was obtained on day 211, 1987 with an exposure time of 1054 minutes. The exposure was begun and terminated during contiguous half Us 2 shifts with approximately 16 hours of intervening ESA and US1 time. The second image SWP33681 was obtained on day 152, 1988 with an exposure time of 755 minutes. This observation was accomplished without contiguous half US2 time. Before each exposure, a low resolution SWP image of GD358 was taken to verify centering.

The spectroscopic data was analyzed with the NASA Goddard Regional Data Analysis Facility (RDAF) software via a phone link between the vax 8350 and the Department of Astronomy and Astrophysics at Villanova University where a Tektronix 4014-1 terminal and Tektronix 4611 copier were utilized for the measurements and display.

III. Results and Summary

The first image SWP31432 was examined in the echelle orders containing the strongest ion transitions of $\mathrm{C}, \mathrm{N}, \mathrm{O}, \mathrm{si}$ and in the wavelength region of He II. This spectrum revealed the presence of numerous interstellar absorption lines identified in table 1 . The velocity of the local interstellar medium (LISM) in the direction to GD358 was found to be $-27 \mathrm{~km} / \mathrm{s}$. In addition absorption features identified as C II (1335.7077) and He II (1640.474) with an equivalent widths of $150 \mathrm{~mA}$ and $250 \mathrm{~mA}$ respectively, were found to be redshifted with respect to the LISM by $34 \mathrm{~km} / \mathrm{s}$. Close examination of the photowrites revealed no obvious artifacts which could account for the line. The identification of a similar feature at C II (1334.5323) was complicated by a reseaux redward of the interstellar line. Since the detection of redshifted $C$ II and He II was unexpected and provides important data points at high DB 


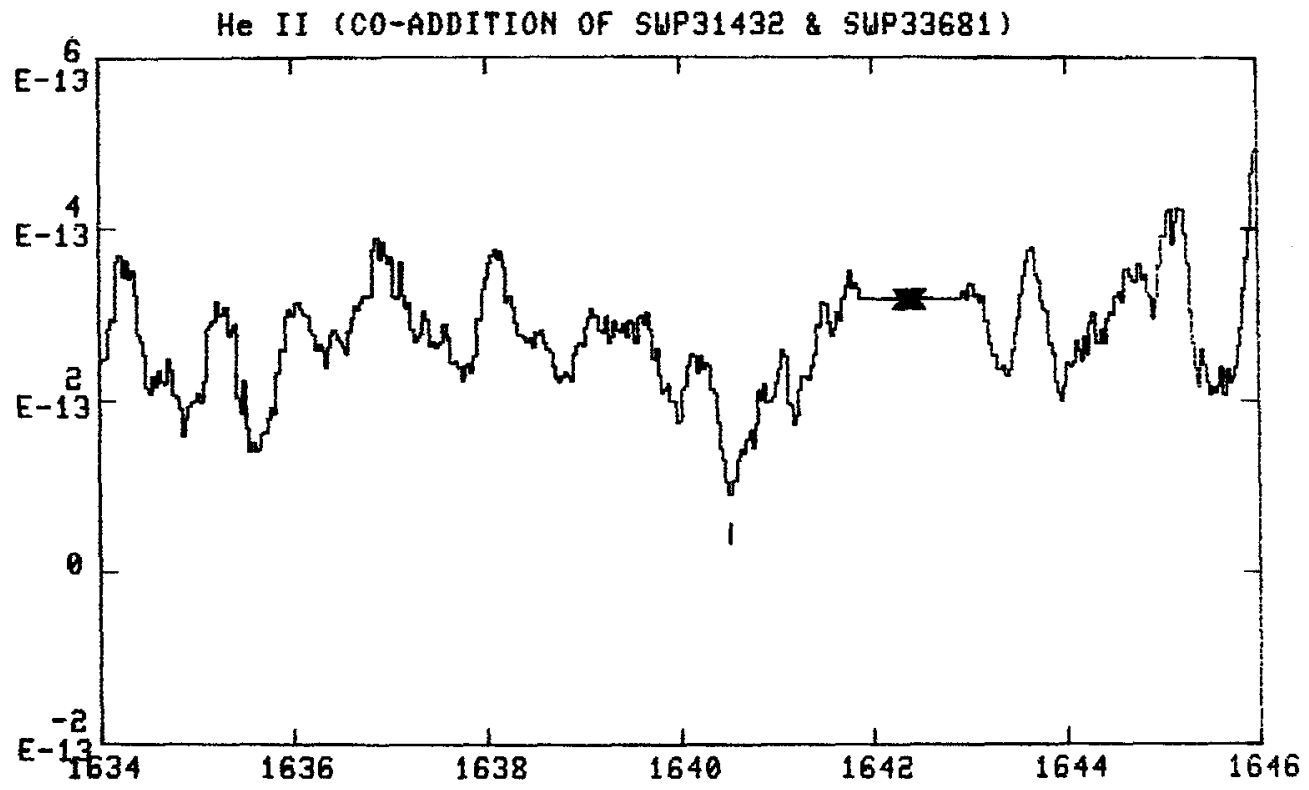

Figure 1----Flux (ordinate, cgs) versus wavelength (abscissa) in Angstroms for the co-added images (SWP31432+SWP33681).

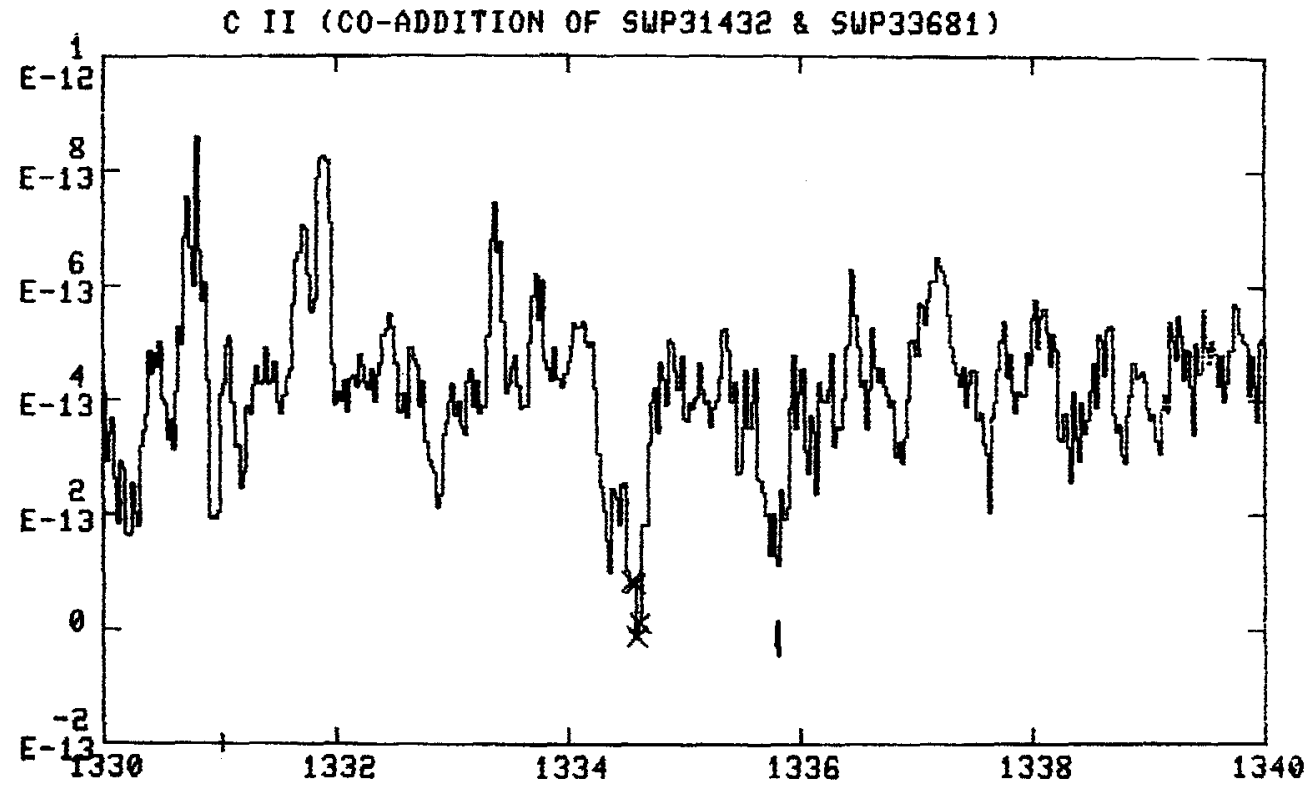

Figure 2.---Same as figure 1 except in the wavelength region of $C$ II. The velocity at the vertical tick mark is $6 \mathrm{~km} / \mathrm{s}$. 
temperatures for tests of convective dredgeup mechanisms (Fontaine et al 1984) and the hot DB temperature scale, a second image was obtained (SWP33681) 10.2 months later in order to confirm the features.

A close examination of the backup image (SWP33681) and comparison with SWP31432 revealed He II and C II at the same redshift $(6-7 \mathrm{~km} / \mathrm{s})$ as in SWP31432, thus confirming the reality of both features. In both spectra, the equivalent width of He II was measured to be approximately $250 \mathrm{~mA}$.

The two images were coadded in the wavelength regions of He II and $C$ II and the results of the coaddition are shown in figures 1 and 2 . Figure $I$ is a flux versus wavelength plot of the He II (1640) coaddition with 10 point smoothing. The feature appears fairly symmetric but with the possible suggestion of multiple components slightly redward and blueward of the central core. These weaker components appear in both individual images. The width of the He II line can be used to place limits on the rotational velocity and magnetic field of GD358 pending a successful model fit to the line profile data in figure 1.

The coaddition of the $C$ II wavelength region is displayed in figure 2 where a flux versus wavelength plot reveals the possible redshifted C II (1335.7077) absorption line with an equivalent width of $134 \mathrm{~mA}$. The central velocity of the line is $6 \mathrm{~km} / \mathrm{s}$, which agrees with the velocity of the He II feature. This velocity coincidence with He II strengthens somewhat, the reality of the $c$ II feature.

The Lyman alpha + He II (1215) region reveals no clear evidence of absorption wings flanking the geocoronal emission. The other echelle orders appear devoid of possible stellar features except for a possible doubled emission feature at Si II with the red side of the emission having a redshift in agreement with He II.

Pending a full model atmosphere analysis now in progress by one of us (G.W.), we can only speculate on the implications of the He II detection. The hot $D B$ temperature scale is presently controversial (cf. Oke, Weidemann and Koester 1984; Liebert et al 1986) with IUE temperature determinations, excluding optical data, yielding significantly higher temperatures for the hottest DB stars and thus a hotter pulsational instability blue edge. The atmospheric parameters of GD358 in particular, were explicitly derived by koester et al. (1985) from high signal to noise spectrophotometry. It is possible that the hot DB temperature scale may now have to be revised upward in 
view of our He II detection. If so, this would favor the higher hot DB temperature scale of Liebert et al. (1986) and could place GD358 in the so-called DO-DB gap $(30000 \mathrm{~K}-45000 \mathrm{~K})$ where to date, no helium-rich degenerates have been found.

If the C II feature is real, the presence of carbon at the effective temperature of GD358 (Teff $=25-28000 \mathrm{~K}$ ), may not be inconsistent with the convective dredgeup theory (Fontaine et al 1984) for the origin of photospheric carbon in cooler helium-rich degenerates. The dredgeup, by helium convection, of carbon from its equilibrium diffusion tail extending upward from the carbon-oxygen core, predicts a low abundance of carbon in the hottest DB stars. It is even possible that alternative explanations for carbon in GD358 may have to be sought perhaps in connection with the non-radial g-mode pulsations of GD358. These possibilities are currently being explored with new diffusion calculations by one of us (G.V.). Encouraged by the unexpected detections reported here for GD358 we plan to obtain one or two additional IUE echelle images of hot DB stars accessible with back to back US-European exposures. Our model atmosphere analysis will be reported in a subsequent paper.

This work was supported by NASA grant NAG5-343 and in part by NSF grant AST88-02689, both to Villanova University and in part by NSF grants AST88-40482 (J.L.) and AST85-15219 (G.W.).

\section{References}

Bruhweiler, F., and Kondo, Y.1983, Ap.J., 269, 657.

Dupree, A., and Raymond, J. Ap.J. (Letters), 263, L63.

Fontaine,G., Villenueve,B., Wesemael, F., and Wegner, G. 1984, Ap.J. (Letters), 277, L51.

Koester, D., Vauclair, G., Dolez, N., Oke, J.B., Greenstein, J.L., and Weidemann, V.1985, Astr. Ap. 149, 423.

Liebert, J., Wesemael, F., Hansen, C.J., Fontaine, G., Shipman, H.L., Sion, E.M., Winget,D., and Green, R.F.1986, Ap.J., 309, 612.

Oke, J.B., Weidemann, V., and Koester, D.1984, Ap.J., 281, 276. Sion, E.M., and Guinan, E.F.1983, Ap.J. (Letters), 265, L87. 